\title{
Significance of Fertility Preservation in Patients Undergoing Cancer Chemotherapy Specifically in Adolescents/Young Adults Requiring Alkylating Agent - A Short Communication
}

\author{
Kulvinder Kochar Kaur ${ }^{1 *}$, Gautam Allahbadia ${ }^{2}$ and Mandeep Singh ${ }^{3}$ \\ ${ }^{1}$ Scientific Director, Dr Kulvinder Kaur Centre for Human Reproduction, Jalandhar, \\ Punjab, India \\ ${ }^{2}$ Scientific Director, Rotunda-A Centre for Human Reproduction, Mumbai, India \\ ${ }^{3}$ Consultant Neurologist, Swami Satyan and Hospital, Jalandhar, Punjab, India \\ *Corresponding Author: Kulvinder Kochar Kaur, Scientific Director, Dr Kulvinder \\ Kaur Centre for Human Reproduction, Jalandhar, Punjab, India.
}

Received: September 15, 2021

Published: September 25, 2021

(C) All rights are reserved by Kulvinder

Kochar Kaur., et al.

\begin{abstract}
With the greater advancements in field of cancer treatment, the survival rates have escalated remarkably, thus the need arising for the fertility preservation in the survivors with regards to oocyte/embryo/ovarian tissue cryopreservation has assumed great importance particularly for those receiving chemotherapy that too with alkylating agents. Furthermore, the need further is enhanced in younger adults along with adolescents diagnosed for cancer and need chemotherapy and are yet to attain puberty where it might not be feasible to do cryopreservation of oocytes. The aim of this short communication is to emphasize on the young cancer patients in whom a diagnosis of cancer is made and which patients stand a chance of generation of diminished ovarian reserve (DOR) who receive chemotherapy as well as the agents that justify evaluation of Antimullerian hormone (AMH) as well as other investigations required prior to initiation of chemotherapy.
\end{abstract}

Keywords: Cancer; Chemotherapy; Alkylating Agents; Fertility Preservation

With the incidence of survival following cancer escalating subsequent to advances in cancer treatment greater incidence of presentation with infertility in addition to premature ovarian insufficiency (POI) is seen [1]. Thus the requirement amongst adolescents in addition to young adults with regards to fertility preservation consciousness along with counseling has escalated side by side. Currently it is not feasible to make anticipation of which patients possess maximum chances of generation of diminished ovarian reserve (DOR), besides the ones that don't. Baseline ovarian reserve (OR) comprises the oocytes that are existent. Antimullerian hormone (AMH) as well as antral follicles count (AFC)by ultrasonography (USG) are markers by proxy for the OR. Chemotherapeutic agents are known to result in infertility via loss of primordial follicles present, besides the kind of agents used, age of exposure in addition to dosages utilized [2]. Woodruff., et al. [3,4], In 2006 itself used the term Oncofertility for which In 2007 they received an award from the National Institute of Health Interdisciplinary Roadmap Grant [5], for the evaluation of this problem [5]. Furthermore, maximum studies with regards to fertility preservation have been done in breast cancer patients with ages in late 30 's/early 40's, thus one cant draw conclusions in younger population with separate diagnosis as well as treatment. The capacity of anticipation of OR in younger patients, would aid in our finding the 
capacity of future reproductive potential, besides gain knowledge with regards to which patients might benefit from fertility preservation [6]. Further we have detailed a lot of articles with regards to oncofertility [7-10].

Moreover, recently Hopeman., et al. [11] tried to associate the clinical variable that were inclusive of age, diagnosis of cancer, besides chemotherapy treatment received In their prospective study. of the 102 postpubertal, female cancer survivors that got recruited in a longitudinal cohort study Hopeman., et al. [11], evaluated the anticipators of diminished ovarian reserve (DOR), which by definition is an antimullerian hormone (AMH) amount that is $<1 \mathrm{ng} / \mathrm{ml}$ that has been estimated $8-24 \mathrm{mth}$ subsequent to chemotherapy stoppage. The study was constituted by patients of age $\geq 30 \mathrm{yrs}$, alkylating agent therapy, breast cancer (vis a vis lymphoma as well as other malignancies in addition to lesser pre treatment AMH possessed an escalation of chances that were correlated with DOR. This particular study again validated that alkylating agents comprised the biggest problem with regards to the future fertility, resulting in 10 times greater chances of DOR following exposure, whose mode of action was presented by us recently [10].

Despite these variables having been determined in retrospective studies, this study gives significant value in view of it being a prospective study. Furthermore, a lot more studies took into account fertility subsequent to cancer therapy, had concentrated on patients with breast cancer possessing an average older age (belonging to late 30's) in contrast to greater divergent oncology population that has been implicated in this study (with average age equivalent to 27yrs) [12]. A drawback of this study is the inclusion criteria not excluding patients with haemorrhagic neoplasms with 42 patients since these patients possess a lesser AMH at the time of manifestation with regards to fertility preservation, due to which another study is required [13].

Finally the biggest observation of this study is that even when younger patient population is there about $80 \%$ of the patients in the study possessed lesser AMH, following chemotherapy. These outcomes corroborate that patients that are about to undergo chemotherapy require counseling, with regards to how fertility preservation becomes essential in view of the fertility problems caused by chemotherapy which warranted a referral to a fertility specialist/ reproductive endocrinologist who is used to these procedures in contrast to the peadiatric oncologist [1]. Besides, that the patients who are not possessing the capacity of making a decision are of importance, in view of the assessment for the particular biomarkers for ovarian reserve (OR) detail, that is either usefulness in addition to restrictions as well as think of fertility preservation or therapy prior to moving further.

The model obtained in this study is distinctive, since it might yield solid answers with regards to patients particularly looking for fertility preservation actually look for- namely individual evaluation, with regards to, evaluation of their chances of DOR subsequent to treatment. Like Hopeman., et al. [11], cited that in their model, a 26yr old patient with lymphoma, a baseline AMH of 6ngml, who would not get therapy with an alkylating agent with regards to chemotherapy possesses just a 5\% probability of DOR following chemotherapy. On confirmation in case of getting offered to big data sets, this model possesses capacity of dynamic calculator, akin to the BWH Egg Freezing Counseling Tool (http://www. mdcalc.com/bwh-egg-freezing-counseling-tool-efct), that could be specifically important for the patients in addition to providers of individualized counseling, (Figure 1).

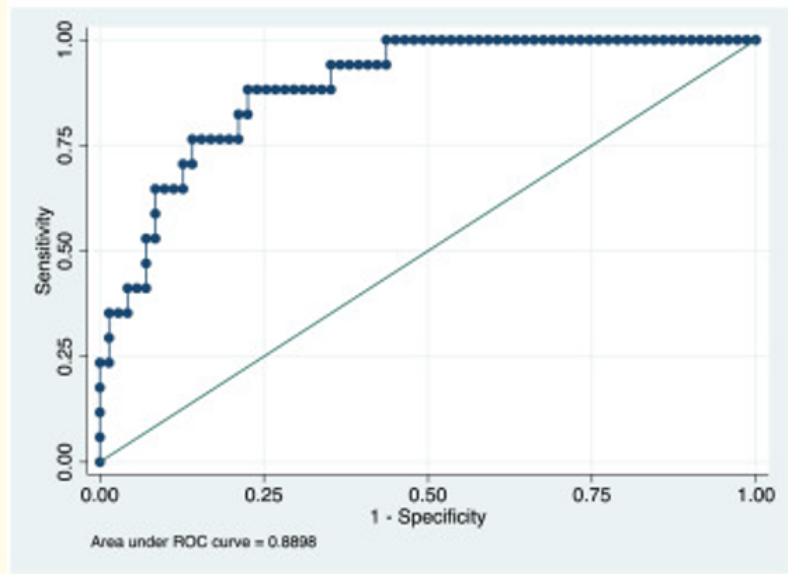

Figure 1: Courtesy ref no-11- Receiver operating characteristic (ROC) curve assessing performance of predictive model of chemotherapy-related diminished ovarian reserve: area under the curve $=0.89(95 \%$ CI 0.83-0.95) .

Moreover, its potential utility beyond the scope of this particular, model might get incorporated with regards to future succession 
in addition to what might give knowledge with regards to fertility preservation decided or not.

1stly, patients needing chemoprophylaxis subsequent to cancer might take into account the future influence, of their primary treatment (that might not be inclusive of chemotherapy, thus lesser chances by the model) besides yrs lost subsequently. In a usual example, young women that present with estrogen receptor (ER) - positive breast cancer get usually counseling to consume tamoxifen for 10yrs, that mostly influences reproductive yrs. Moreover, in case, if any patient decides to stop chemoprophylaxis subsequent to 2-3yrs for pregnancy, while consulting their oncologist, with full insight of this decision that is away from the standard of care, early involving of their, reproductive endocrinologist in addition to, in vitro fertilization (IVF) are usually advocated to escalate, live birth rates (LBR), besides reintroducing chemoprophylaxis. Thus, despite these patients being at lesser risk for treatment - associated DOR, oocyte/embryo cryopreservation needs to be take into account, even now in seriousness having the insight of continuing treatment timeline.

2ndly the way Hopeman., et al. [11], accepted, that this study is neither fashioned nor powered with regards to, assessing radiotherapy, with just 9 patients obtained for radiation therapy. Pelvic radiation therapy is an accepted to be a robust negative anticipator of future reproductive results [14]. Ovarian radiation therapy causes fast loss of the pool of follicles in addition to might be resulting in, POI, while Pelvic radiation might injure the endometrium/ myometrium, resulting in uterine factor infertility as well as/or obstetric complications inclusive of miscarriage as well as preterm birth [14]. Thus Pelvic irradiation that is inclusive of ovaries would become an important variable In case of greater comprehensive, anticipator model.

Moreover, in future both studies in addition to anticipator model in ideal circumstances need to be inclusive of fertility results that possess greater meaning, besides take into account if patients carry a diagnosis of clinical in fertility or attempt a live birth. This is a much tougher result to attain as cited by Hopeman., et al. [11], in view of lack of survival of a lot of patients in addition to many might not try to attain a pregnancy if anytime ever in future [15].

\section{Conclusions}

With the documentation of a greater chances of DOR in distinctive oncology population, this study makes it necessary, for fertility preservation, consultation for each patient from reproductive age who require chemotherapy treatment, Hence patients needing cancer therapy have to decide fast with regards to the complicated issue of their future fertility. In such instances, which possess sensitivity, model like this one (as well as further versions that have embedded post treatment prophylaxis, radiation therapy in addition to birth results) would aid clinicians to give individualized counseling to make the patients empowered for taking informed decision making that suits their future life.

\section{Bibliography}

1. Ethics Committee of the American Society of Reproductive Medicine. "Fertility preservation and reproduction in patients facing gonadotoxic therapies: an Ethics Committee opinion". Fertility and Sterility 110 (2018): 380-386.

2. Such., et al. "Pre chemotherapy antimullerian hormone, age and body size predict timing of return of ovarian function in young breast patients". Cancer 120 (2014): 3691-3698.

3. Woodruff TK., et al. "A view from the past into our collective future: the Oncofertility consortium vision statement". Journal of Assisted Reproduction and Genetics 38 (2021): 3-15.

4. Anazodo A., et al. "How can we improve oncofertility care for patients? A systematic scoping review of current international practice and models of care". Human Reproduction Update 25.2 (2019): 159-179.

5. Smith BM., et al. "The National Physicians Cooperative transforming fertility management in the cancer setting and beyond". Future Oncology 14 (2018): 3059-3072.

6. Vogt KS., et al. "Preserving Fertility in women with Cancer (PreFer) decision making and patientsreported outcomes in women offered egg and embryo banking prior to Cancer treatment". Psychooncology 27 (2018): 2725-2732.

7. Kulvinder Kochar Kaur., et al. "An Update on Management of Oncofertility-Does the Use of Vsels Appear Practical in the Near Future in Human Malignancies Replacing Cortical Tissue/Tes- 
ticular Tissue Transplantation". International Journal of Stem Cells and Regenerative Medicine 1.1 (2019): 103.

8. Kulvinder Kochar Kaur., et al. "How Far Have We Reached with Regards to Our Endeavours in Testicular Tissue Transplantation Along with in Vitro Spermatogenesis After Success in Animals - A Systematic Review". Archives of Urology 3.12 (2020): $1-15$.

9. Kulvinder Kochar Kaur., et al. "An update on fertility preservation in men undergoing cancer treatment, irrespective of age-A Comprehensive Review".

10. Kulvinder Kochar Kaur., et al. "The mechanism by which chemotherapy with use of alkylating agents cause follicular activation: is there any further mode for the loss of the primordial follicles? A Short Communication". Journal of Gynecology 6.3 (2021): 000222.

11. Hopeman MM., et al. "A predictive model for chemotherapy related diminished ovarian reserve in reproductive aged women". Fertility and Sterility 115 (2021): 431-437.

12. Moore HC., et al. "Goserelin for ovarian protection during breast Cancer adjuvant cancer therapy". The New England Journal of Medicine 372 (2015): 923-932.

13. Mehm AR., et al. "Fertility preservation in marrow failure syndrome prior to allogenic stem cells transplantation". American Journal of Haematology 9372 (2018): E190-193.

14. Meirow D., et al. "Toxicity of chemotherapy and radiation on female reproduction". Clinical Obstetrics and Gynecology 53 (2020): 727-739.

15. Brady PC and Forman EJ. "An Oncofertility prediction tool? Forecasting, fertility after Cancer". Fertility and Sterility 115

(2021): 323-324.

\section{Volume 5 Issue 10 October 2021}

(C) All rights are reserved by Kulvinder Kochar Kaur.,

et al. 